International Electronic Journal of Algebra

Volume 31 (2022) 74-89

DOI: $10.24330 /$ ieja.1058417

\title{
ON S-PRIMARY SUBMODULES
}

\author{
H. Ansari-Toroghy and S. S. Pourmortazavi
}

Received: 16 October 2020; Revised: 4 May 2021; Accepted: 5 May 2021

Communicated by Abdullah Harmancı

\begin{abstract}
Let $R$ be a commutative ring with identity, $S$ a multiplicatively closed subset of $R$, and $M$ be an $R$-module. In this paper, we study and investigate some properties of $S$-primary submodules of $M$. Among the other results, it is shown that this class of modules contains the family of primary (resp. $S$-prime) submodules properly.
\end{abstract}

Mathematics Subject Classification (2020): 13C13, 13C05, 13A15

Keywords: Multiplicatively closed subset, prime submodule, $S$-prime submodule, primary submodule, $S$-primary submodule

\section{Introduction}

Throughout this article, all rings are commutative with identity elements and all modules are unital modules. $\mathbb{N}, \mathbb{Z}$, and $\mathbb{Q}$ will denote respectively the natural numbers, the ring of integers and the field of quotients of $\mathbb{Z}$.

Consider a non-empty subset $S$ of $R$. We call $S$ a multiplicatively closed subset of $R$ if (i) $0 \notin S$, (ii) $1 \in S$, and (iii) $s s^{\prime} \in S$ for all $s, s^{\prime} \in S$ [12]. Note that $S=R-p$ is a multiplicatively closed subset of $R$ for every prime ideal $p$ of $R$. Let $N$ and $K$ be two submodules of an $R$-module $M$ and $J$ an ideal of $R$. Then the residual $N$ by $K$ and $J$ is defined as follows:

$$
\begin{gathered}
\left(N:_{R} K\right)=\{r \in R \mid r K \subseteq N\}, \\
\left(N:_{M} J\right)=\{m \in M \mid J m \subseteq N\} .
\end{gathered}
$$

Particularly, we use $A n n_{R}(M)$ instead of $\left(0:_{R} M\right)$ and $\left(N:_{M} s\right)$ instead of $\left(N:_{M} R s\right)$, where $R s$ is the principal ideal generated by an element $s \in R$. The sets of prime ideals and maximal ideals of $R$ are denoted by $\operatorname{Spec}(R)$ and $\operatorname{Max}(R)$, respectively.

A submodule $P$ of $M$ is called prime if $P \neq M$ and whenever $r \in R$ and $e \in M$ satisfy $r e \in P$, then $r \in\left(P:_{R} M\right)$ or $e \in P$. The set of all prime submodules of $M$ is denoted by $\operatorname{Spec}(M)[3,7]$.

In [11], the authors introduced the concept of $S$-prime submodules and investigated some properties of this class of modules. Let $S$ be a multiplicatively closed 
subset of $R$ and $P$ be a submodule of $M$ with $\left(P:_{R} M\right) \cap S=\emptyset$. Then $P$ is said to be an $S$-prime submodule if there exists $s \in S$ such that whenever $r m \in P$, where $r \in R$ and $m \in M$, then $s r \in\left(P:_{R} M\right)$ or $s m \in P$. Particularly, an ideal $I$ of $R$ is said to be an $S$-prime ideal if $I$ is an $S$-prime submodule of the $R$-module $R$.

The notion of $S$-primary submodule was introduced in [5]. Let $S$ be a multiplicatively closed subset of $R$ and $P$ be a submodule of $M$ with $\left(P:_{R} M\right) \cap S=\emptyset$. Then $P$ is said to be an $S$-primary submodule if there exists $s \in S$ such that whenever $r m \in P$, where $r \in R$ and $m \in M$, then $s r \in \sqrt{\left(P:_{R} M\right)}$ or $s m \in P$.

In this paper, we will study the family of $S$-primary submodules extensively and investigate some of their properties. In fact, this family of modules is a generalization of primary (resp. $S$-prime) submodules.

Among the other results, we provide some notions that each one is equivalent to $S$-primary (Theorem 2.2). Examples 2.4 and 2.5 show that these new modules contain the family of primary and $S$-prime submodules properly. Further it is proved that if $P$ is an $S$-primary submodule of $M$, then $S^{-1} P$ is also an $S$-primary submodule of $S^{-1} M$ (Proposition 2.7). Example 2.8 shows that the converse is not true in general. Also we show that $S$-primary submodules has a good behavior with Cartesian products (Theorems 2.20 and 2.21). Moreover, we provide some useful characterization concerning $S$-primary submodules (Theorems 2.17, 2.24 and 2.25).

\section{Main results}

Definition 2.1. Let $S$ be a multiplicatively closed subset of $R$ and $P$ be a submodule of $M$ with $\left(P:_{R} M\right) \cap S=\emptyset$. Then $P$ is said to be an $S$-primary submodule of $M$ if there exists $s \in S$ such that whenever $r m \in P$, where $m \in M$ and $r \in R$, then $s r \in \sqrt{\left(P:_{R} M\right)}$ or $s m \in P$ [5, Definition 2.27]. In particular, we say that an ideal $I$ of $R$ is an $S$-primary ideal if $I$ is an $S$-primary submodule of $R$-module $R$.

Theorem 2.2. Let $S$ be a multiplicatively closed subset of $R$. For a submodule $P$ of an $R$-module $M$ with $\left(P:_{R} M\right) \cap S=\emptyset$, the following are equivalent:

(a) $P$ is an $S$-primary submodule of $M$;

(b) There exists $s \in S$ such that for every $r \in R$, the endomorphism $r: s(M / P) \rightarrow s(M / P)$ given by $s \bar{m}=s m+P \mapsto r s \bar{m}=r s m+P$ is injective or $(r s)^{t}(M / P)=(\overline{0})$ for some $t \in \mathbb{N}$;

(c) There exists $s \in S$ such that whenever $r \subseteq P$, where $N$ is a submodule of $M$ and $r \in R$, then $s r \in \sqrt{\left(P:_{R} M\right)}$ or $s N \subseteq P$;

(d) There exists $s \in S$ such that whenever $J N \subseteq P$, where $N$ is a submodule of $M$ and $J$ is an ideal of $R$, then $s J \subseteq \sqrt{\left(P:_{R} M\right)}$ or $s N \subseteq P$. 
Proof. (a) $\Rightarrow$ (b) By hypothesis, there exists $s \in S$ such that for every $r \in R$ and $m \in M$ if $r m \in P$, then we have $s m \in P$ or $s r \in \sqrt{\left(P:_{R} M\right)}$. Now for each $r \in R$, we define the endomorphism $r: s(M / P) \rightarrow s(M / P)$ by $s m+P \mapsto r s m+P$. We show that this endomorphism is injective or $r s \in \sqrt{\left(P:_{R} M\right)}$. Assume $r s \notin$ $\sqrt{\left(P:_{R} M\right)}$. Then we show the other part holds. To see let $\overline{r s m}=r s m+P=P=$ $\overline{0}$. So we have $(r s) m \in P$. So by hypothesis, $s m \in P$ or $s(r s)=r s^{2} \in \sqrt{\left(P:_{R} M\right)}$. We conclude $s r \in \sqrt{\left(P:_{R} M\right)}$, which is a contradiction. Hence $s m \in P$, as required.

(b) $\Rightarrow$ (a) It is clear.

(a) $\Rightarrow$ (c) It is clear.

(c) $\Rightarrow$ (d) Let $J N \subseteq P$, where $J$ is an ideal of $R$ and $N$ is a submodule of $M$. We will show that there exists $s \in S$ such that $s N \subseteq P$ or $s J \subseteq \sqrt{\left(P:_{R} M\right)}$. Clearly, we have $r N \subseteq P$ for every $r \in J$. So by part (c), there exists $s \in S$ such that $s N \subseteq P$ or $s r \in \sqrt{\left(P:_{R} M\right)}$ for every $r \in J$, as desired.

(d) $\Rightarrow$ (a) Take $r \in R$ and $m \in M$ with $r m \in P$. Now, put $J=R r$ and $N=R m$. Then we can conclude that $J N=R r m \subseteq P$. By assumption, there is an $s \in S$ so that $s J=R r s \subseteq \sqrt{\left(P:_{R} M\right)}$ or $s N=R s m \subseteq P$ and so either $s r \in \sqrt{\left(P:_{R} M\right)}$ or $s m \in P$, as required.

Lemma 2.3. Let $M$ be an $R$-module and $S$ a multiplicatively closed subset of $R$. Then we have the following.

(a) If $P$ is a primary submodule of $M$ such that $\left(P:_{R} M\right) \cap S=\emptyset$, then $P$ is an $S$-primary submodule of $M$.

(b) If $P$ is an $S$-primary submodule of $M$ and $S \subseteq u(R)$, where $u(R)$ denotes the set of units in $R$, then $P$ is a primary submodule of $M$.

Proof. This is clear.

By setting $S=\{1\}$, we conclude that every primary submodule is an $S$-primary submodule by Lemma 2.3. The following example shows that the converse is not true in general.

Example 2.4. Consider the $\mathbb{Z}$-module $M=\mathbb{Q} \oplus\left(\bigoplus_{i=1}^{n} \mathbb{Z}_{p_{i}}\right)$, where $p_{i}$ are distinct positive prime integers. Take the submodule $P=(0)$ and the multiplicatively closed subset

$$
S=\left\{1, p_{1}^{m_{1}} p_{2}^{m_{2}} \ldots p_{n}^{m_{n}} \mid \forall i \in\{1,2, \ldots, n\}, m_{i} \in \mathbb{N} \cup\{0\}\right\}
$$

First note that $\left(P:_{\mathbb{Z}} M\right)=(0)$ and $p_{1} p_{2} \ldots p_{n}(0, \overline{1}, \overline{1}, \ldots, \overline{1})=(0, \overline{0}, \overline{0}, \ldots, \overline{0}) \in P$. Since $p_{1} p_{2} \ldots p_{n} \notin \sqrt{(P: \mathbb{Z} M)}$ and $(0, \overline{1}, \overline{1}, \ldots, \overline{1}) \notin P, P$ is not a primary submodule 
of $M$. Put $s=p_{1} p_{2} \ldots p_{n}$ and let

$$
k\left(\frac{a}{b}, \overline{x_{1}}, \overline{x_{2}}, \ldots, \overline{x_{n}}\right)=\left(\frac{k a}{b}, \overline{k x_{1}}, \overline{k x_{2}}, \ldots, \overline{k x_{n}}\right) \in P,
$$

where $k \in \mathbb{Z}$ and $\left(\frac{a}{b}, \overline{x_{1}}, \overline{x_{2}}, \ldots, \overline{x_{n}}\right) \in M$. Then $k a=0$. This yields that $k=0$ or $a=0$. If $k=0$, there is nothing to prove. Thus assume that $a=0$. Then $s\left(\frac{a}{b}, \overline{x_{1}}, \overline{x_{2}}, \ldots, \overline{x_{n}}\right) \in P$. Therefore, $P$ is an $S$-primary submodule of $M$.

We recall that a submodule $P$ of an $R$-module $M$ is $S$-prime if there exists $s \in S$ such that whenever $r m \in P$, where $r \in R$ and $m \in M$, then $s r \in\left(P:_{R} M\right)$ or $s m \in P$ [11]. Clearly, every $S$-prime submodule is $S$-primary. The following example shows that the converse is not true in general.

Example 2.5. Consider $M=\mathbb{Z}_{4}$ as a $\mathbb{Z}$-module. Set $S=\mathbb{Z} \backslash 2 \mathbb{Z}$ and $P=(\overline{0})$. Thus we have $\left(P:_{\mathbb{Z}} M\right)=4 \mathbb{Z}$ and $2 . \overline{2} \in(\overline{0})$. Since for every $s \in S, 2 s \notin\left(P:_{\mathbb{Z}} M\right)$ and $s . \overline{2} \notin P, P$ is not an $S$-prime submodule of $M$. Put $s=1$ and let $k \bar{a}=\overline{0}$. If $\bar{a}=\overline{0}$, there is nothing to prove. Thus assume that $\bar{a} \neq \overline{0}$. Then $k=2 k^{\prime}$ for some $k^{\prime} \in \mathbb{Z}$. This implies that $k \in \sqrt{\left(P:_{\mathbb{Z}} M\right)}$. Therefore, $P$ is an $S$-primary submodule of $M$.

Remark 2.6. Let $S$ be a multiplicatively closed subset of $R$. Recall that the saturation $S^{*}$ of $S$ is defined as

$$
S^{*}=\left\{x \in R \mid \frac{x}{1} \text { is a unit of } S^{-1} R\right\} .
$$

It is obvious that $S^{*}$ is a multiplicatively closed subset of $R$ containing $S$ [6].

Proposition 2.7. Let $S$ be a multiplicatively closed subset of $R$ and $M$ be an $R$-module. Then we have the following.

(a) If $S_{1} \subseteq S_{2}$ are multiplicatively closed subsets of $R$ and $P$ is an $S_{1}$-primary submodule of $M$, then $P$ is an $S_{2}$-primary submodule of $M$ in case $\left(P:_{R} M\right) \cap S_{2}=\emptyset$.

(b) $P$ is an $S$-primary submodule of $M$ if and only if $P$ is an $S^{*}$-primary submodule of $M$.

(c) If $P$ is an $S$-primary submodule of $M$, then $S^{-1} P$ is a primary submodule of $S^{-1} R$-module $S^{-1} M$.

Proof. (a) It is clear.

(b) Assume that $P$ is an $S$-primary submodule of $M$. We need to prove that $\left(P:_{R} M\right)$ and $S^{*}$ are disjoint. Suppose there exists $x \in\left(P:_{R} M\right) \cap S^{*}$. As $x \in S^{*}, \frac{x}{1}$ is a unit of $S^{-1} R$ and so $\left(\frac{x}{1}\right)\left(\frac{a}{s}\right)=1$ for some $a \in R$ and $s \in S$. This yields that $u s=u x a$ for some $u \in S$. Now we have that $u s=u x a \in$ $\left(P:_{R} M\right) \cap S$, a contradiction. Thus $\left(P:_{R} M\right) \cap S^{*}=\emptyset$. Now as $S \subseteq S^{*}$, by 
part (a), $P$ is an $S^{*}$-primary submodule of $M$. Conversely, assume that $P$ is an $S^{*}$-primary submodule of $M$. Let $r m \in P$, where $r \in R$ and $m \in M$. Then there exists $x \in S^{*}$ such that $x r \in \sqrt{\left(P:_{R} M\right)}$ or $x m \in P$. As $\frac{x}{1}$ is a unit of $S^{-1} R$, there exist $u, s \in S$ and $a \in R$ such that $u s=u x a$. Put $u s=s^{\prime} \in S$. Then note that $s^{\prime} r=(u s) r=u a x r \in \sqrt{\left(P:_{R} M\right)}$ or $s^{\prime} m \in P$. Therefore, $P$ is an $S$-primary submodule of $M$.

(c) Let $\left(\frac{r}{s}\right)\left(\frac{m}{t}\right) \in S^{-1} P$, where $\frac{r}{s} \in S^{-1} R$ and $\frac{m}{t} \in S^{-1} M$. Then urm $\in P$ for some $u \in S$. Since $P$ is an $S$-primary submodule of $M$, there is an $s^{\prime} \in S$ so that $s^{\prime} u r \in \sqrt{\left(P:_{R} M\right)}$ or $s^{\prime} m \in P$. This yields that $\frac{r}{s}=\frac{s^{\prime} u r}{s^{\prime} u s} \in$ $S^{-1} \sqrt{\left(P:_{R} M\right)} \subseteq \sqrt{\left(S^{-1} P:_{S^{-1} R} S^{-1} M\right)}$ or $\frac{m}{t}=\frac{s^{\prime} m}{s^{\prime} t} \in S^{-1} P$. Hence, $S^{-1} P$ is a primary submodule of $S^{-1} M$.

The following example shows that the converse of part (c) of Proposition 2.7 is not true in general.

Example 2.8. Consider the $\mathbb{Z}$-module $M=\mathbb{Q}$. Take the submodule $N=\mathbb{Z}$ and the multiplicatively closed subset $S=\mathbb{Z}-\{0\}$ of $\mathbb{Z}$. Then $\left(N:_{\mathbb{Z}} M\right)=(0)$. Let $s$ be an arbitrary element of $S$. Choose a prime number $p$ with $\operatorname{gcd}(p, s)=1$. Then note that $p \frac{1}{p}=1 \in N$. But $s p \notin \sqrt{\left(N:_{\mathbb{Z}} M\right)}$ and $\frac{s}{p} \notin N$, it follows that $N$ is not an $S$-primary submodule of $M$. Since $S^{-1} \mathbb{Z}=\mathbb{Q}$ is a field, $S^{-1}(\mathbb{Q})$ is a vector space. Therefore the proper submodule $S^{-1} N$ is a primary submodule of $S^{-1} \mathbb{Q}$.

Proposition 2.9. Suppose $f: M \rightarrow M^{\prime}$ is an $R$-homomorphism. Then we have the following.

(a) If $P^{\prime}$ is an S-primary submodule of $M^{\prime}$ provided that $\left(f^{-1}\left(P^{\prime}\right):_{R} M\right) \cap S=$ $\emptyset$, then $f^{-1}\left(P^{\prime}\right)$ is an $S$-primary submodule of $M$.

(b) If $f$ is an epimorphism and $P$ is an $S$-primary submodule of $M$ with $\operatorname{ker}(f) \subseteq P$, then $f(P)$ is an $S$-primary submodule of $M^{\prime}$.

Proof. (a) Let $r m \in f^{-1}\left(P^{\prime}\right)$ for some $r \in R$ and $m \in M$. This yields that $f(r m)=r f(m) \in P^{\prime}$. Since $P^{\prime}$ is an $S$-primary submodule of $M^{\prime}$, there is an $s \in S$ so that $s r \in \sqrt{\left(P^{\prime}:_{R} M^{\prime}\right)}$ or $s f(m) \in P^{\prime}$. Now we will show that $\left(P^{\prime}:_{R} M^{\prime}\right) \subseteq\left(f^{-1}\left(P^{\prime}\right):_{R} M\right)$. Take $x \in\left(P^{\prime}:_{R} M^{\prime}\right)$. Then we have $x M^{\prime} \subseteq P^{\prime}$. Since $f(M) \subseteq M^{\prime}$, we conclude that $f(x M)=$ $x f(M) \subseteq x M^{\prime} \subseteq P^{\prime}$. This implies that $x M \subseteq f^{-1}(f(M)) \subseteq f^{-1}\left(p^{\prime}\right)$ and thus $x \in\left(f^{-1}\left(P^{\prime}\right):_{R} M\right)$. Hence we have $s r \in \sqrt{\left(f^{-1}\left(P^{\prime}\right):_{R} M\right)}$ or $s m \in f^{-1}\left(P^{\prime}\right)$. It follows that $f^{-1}\left(P^{\prime}\right)$ is an $S$-primary submodule of $M$.

(b) First note that $\left(f(P):_{R} M^{\prime}\right) \cap S=\emptyset$. Otherwise there would be an $s \in\left(f(P):_{R} M^{\prime}\right) \cap S$. Since $s \in\left(f(P):_{R} M^{\prime}\right), s M^{\prime} \subseteq f(P)$, but then 
$f(s M)=s f(M)=s M^{\prime} \subseteq f(P)$. By taking their inverse images under $f$, we have

$$
s M \subseteq s M+k e r(f) \subseteq f^{-1}(f(P))=P+\operatorname{ker}(f)=P .
$$

That means $s \in\left(P:_{R} M\right)$, which is a contradiction. Now take $r \in R$ and $m^{\prime} \in M^{\prime}$ with $r m^{\prime} \in f(P)$. As $f$ is an epimorphism, there is an $m \in M$ such that $m^{\prime}=f(m)$. Then $r m^{\prime}=r f(m)=f(r m) \in f(P)$. Since $\operatorname{Ker}(f)$ is a subset of $P$, we get $r m \in P$. As $P$ is an $S$-primary submodule of $M$, there is an $s \in S$ so that $s r \in \sqrt{\left(P:_{R} M\right)}$ or $s m \in P$. Since $\sqrt{\left(P:_{R} M\right)} \subseteq \sqrt{\left(f(P):_{R} M^{\prime}\right)}$, we have $s r \in \sqrt{\left(f(P):_{R} M^{\prime}\right)}$ or $f(s m)=$ $s f(m)=s m^{\prime} \in f(P)$. Accordingly, $f(P)$ is an $S$-primary submodule of $M^{\prime}$.

Corollary 2.10. Let $S$ be a multiplicatively closed subset of $R$ and take a submodule $L$ of $M$. Then we have the following.

(a) If $P^{\prime}$ is an $S$-primary submodule of $M$ with $\left(P^{\prime}:_{R} L\right) \cap S=\emptyset$, then $L \cap P^{\prime}$ is an $S$-primary submodule of $L$.

(b) Suppose that $P$ is a submodule of $M$ with $L \subseteq P$. Then $P$ is an $S$-primary submodule of $M$ if and only if $P / L$ is an $S$-primary submodule of $M / L$.

Proof. (a) Consider the injection $i: L \rightarrow M$ defined by $i(m)=m$ for all $m \in L$. Then note that $i^{-1}\left(P^{\prime}\right)=L \cap P^{\prime}$. Now we will show that $\left(i^{-1}\left(P^{\prime}\right):_{R}\right.$ $L) \cap S=\emptyset$. Assume that $s \in\left(i^{-1}\left(P^{\prime}\right):_{R} L\right) \cap S$. Then we have $s L \subseteq$ $i^{-1}\left(P^{\prime}\right)=L \cap P^{\prime} \subseteq P^{\prime}$. This implies that $s \in\left(P^{\prime}:_{R} L\right) \cap S$, a contradiction. The rest follows from Proposition 2.9 (a).

(b) Assume that $P$ is an $S$-primary submodule of $M$. Then consider the canonical homomorphism $\pi: M \rightarrow M / L$ defined by $\pi(m)=m+L$ for all $m \in M$. By Proposition 2.9 (b), $P / L$ is an $S$-primary submodule of $M / L$. Conversely, assume that $P / L$ is an $S$-primary submodule of $M / L$. Let $r m \in P$ for some $r \in R$ and $m \in M$. This yields that $r(m+L)=r m+L \in P / L$. As $P / L$ is an $S$-primary submodule of $M / L$, there is an $s \in S$ so that $s r \in \sqrt{\left(P / L:_{R} M / L\right)}=\sqrt{\left(P:_{R} M\right)}$ or $s(m+L)=s m+L \in P / L$. Therefore, we have $s r \in \sqrt{\left(P:_{R} M\right)}$ or $s m \in P$. Hence, $P$ is an $S$-primary submodule of $M$.

An $R$-module $M$ is said to be a multiplication module if for every submodule $N$ of $M$ there exists an ideal $I$ of $R$ such that $N=I M[4]$.

Proposition 2.11. Let $M$ be an $R$-module and $S$ be a multiplicatively closed subset of $R$. The following statements hold. 
(a) If $P$ is an $S$-primary submodule of $M$, then $\left(P:_{R} M\right)$ is an $S$-primary ideal of $R$.

(b) If $M$ is a multiplication module and $\left(P:_{R} M\right)$ is an $S$-primary ideal of $R$, then $P$ is an $S$-primary submodule of $M$.

Proof. (a) Let $x y \in\left(P:_{R} M\right)$ for some $x, y \in R$. Then $x y m \in P$ for all $m \in M$. As $P$ is an $S$-primary submodule, there exists $s \in S$ such that $s x \in \sqrt{\left(P:_{R} M\right)}$ or sym $\in P$ for all $m \in M$. If $s x \in \sqrt{\left(P:_{R} M\right)}$, there is nothing to prove. Suppose that $s x \notin \sqrt{\left(P:_{R} M\right)}$. Then sym $\in P$ for all $m \in M$ so that $s y \in\left(P:_{R} M\right)$. Therefore, $\left(P:_{R} M\right)$ is an $S$-primary ideal of $R$.

(b) Let $J$ be an ideal of $R$ and $N$ a submodule of $M$ with $J N \subseteq P$. Then we can conclude that $J\left(N:_{R} M\right) \subseteq\left(J N:_{R} M\right) \subseteq\left(P:_{R} M\right)$. As $\left(P:_{R} M\right)$ is an $S$-primary ideal of $R$, there is an $s \in S$ so that $s\left(N:_{R} M\right) \subseteq\left(P:_{R} M\right)$ or $s J \subseteq \sqrt{\left(P:_{R} M\right)}$. Thus, we can conclude that $s N=s\left(N:_{R} M\right) M \subseteq$ $\left(P:_{R} M\right) M=P$ or $s J \subseteq \sqrt{\left(P:_{R} M\right)}$. Therefore, by Theorem $2.2(\mathrm{~d}), P$ is an $S$-primary submodule of $M$.

Remark 2.12. $\quad$ (a) Assume that $M$ is a multiplication $R$-module and $K, L$ are two submodules of $M$. The product of $K$ and $L$ is defined as $K L=\left(K:_{R}\right.$ $M)\left(L:_{R} M\right) M[1]$.

(b) Let $M$ be an $R$-module and $N$ a submodule of $M$. The radical of $N$, denoted by $\operatorname{rad}(N)$, is the intersection of all prime submodules of $M$ containing $N$; that is, $\operatorname{rad}(N)=\bigcap\{P \mid N \subseteq P, P \in \operatorname{Spec}(M)\}[8]$.

As an immediate consequence of the Proposition 2.11 and Theorem 2.2 (d), we have the following explicit result.

Corollary 2.13. Suppose that $M$ is a multiplication $R$-module and $P$ a submodule of $M$ provided that $\left(P:_{R} M\right) \cap S=\emptyset$, where $S$ is a multiplicatively closed subset of $R$. Then the following are equivalent:

(a) $P$ is an $S$-primary submodule of $M$;

(b) There exists $s \in S$ such that whenever $L N \subseteq P$, where $L$ and $N$ are submodules of $M$, then $s\left(L:_{R} M\right) \subseteq \sqrt{\left(P:_{R} M\right)}$ or $s N \subseteq P$.

Corollary 2.14. Suppose that $M$ is a finitely generated multiplication $R$-module and $P$ is a submodule of $M$ provided that $\left(P:_{R} M\right) \cap S=\emptyset$, where $S$ is a multiplicatively closed subset of $R$. Then the following are equivalent:

(a) $P$ is an $S$-primary submodule of $M$; 
(b) There exists $s \in S$ such that whenever $L N \subseteq P$, where $L$ and $N$ are submodules of $M$, then $s L \subseteq \operatorname{rad}(P)$ or $s N \subseteq P$.

Proof. $(\mathrm{a}) \Rightarrow(\mathrm{b})$ Assume that $L N \subseteq P$, where $L$ and $N$ are submodules of $M$. By Remark $2.12(\mathrm{a}), L N=\left(L:_{R} M\right) N \subseteq P$. Then there exists $s \in S$ so that $s\left(L:_{R} M\right) \subseteq \sqrt{\left(P:_{R} M\right)}$ or $s N \subseteq P$ by Theorem $2.2(\mathrm{~d})$. Since $M$ is multiplication, by $\left[4\right.$, Theorem 2.12], we have $s\left(L:_{R} M\right) M=s L \subseteq \sqrt{\left(P:_{R} M\right)} M=\operatorname{rad}(P)$ or $s N \subseteq P$.

(b) $\Rightarrow$ (a) Assume that $J N \subseteq P$, where $N$ is a submodule of $M$ and $J$ is an ideal of $R$. Set $K:=J M$. As $M$ is a multiplication module, Then we have

$$
K N=\left(K:_{R} M\right)\left(N:_{R} M\right) M=J\left(N:_{R} M\right) M=J N \subseteq P .
$$

By assumption, there exists $s \in S$ so that $s K \subseteq \operatorname{rad}(P)$ or $s N \subseteq P$. As $M$ is finitely generated, by [9, Thoerem 4.4], $s K \subseteq \operatorname{rad}(P)$ implies that

$$
s J \subseteq\left(s K:_{R} M\right) \subseteq\left(\operatorname{rad}(P):_{R} M\right)=\sqrt{\left(P:_{R} M\right)} .
$$

Therefore $P$ is an $S$-primary submodule of $M$ by Corollary 2.13.

Remark 2.15. (a) Let $M$ be an $R$-module and $p$ be a maximal ideal of $R$. In [4], $T_{p}(M)$ is defined as follows

$$
T_{p}(M)=\{m \in M \mid(1-r) m=0 \text { for some } r \in p\} .
$$

Clearly $T_{p}(M)$ is a submodule of $M$. An $R$-module $M$ is said to be $p$-cyclic provided there exist $q \in p$ and $m \in M$ such that $(1-q) M \subseteq R m$ [4].

(b) Let $M$ be an $R$-module. Then $M$ is a multiplication $R$-module if and only if for every maximal ideal $p$ of $R$ either $M=T_{p}(M)$ or $M$ is $p$-cyclic [4, Theorem 1.2].

Lemma 2.16. Let $S$ be a multiplicatively closed subset of $R, p$ be an $S$-primary (resp. S-prime) ideal of $R$ and $M$ be a faithful multiplication $R$-module. Then there exists an $s \in S$ such that whenever am $\in p M$, where $a \in R$ and $m \in M$, then $s a \in \sqrt{p}$ (resp. sa $\in$ p) or $s m \in p M$.

Proof. It is enough to prove it for $S$-primary submodules. The technique is similar for $S$-prime. As $p$ is an $S$-primary ideal, there exists $s \in S$, whenever $r r^{\prime} \in p$, where $r, r^{\prime} \in R$, then $s r \in \sqrt{p}$ or $s r^{\prime} \in p$. Let $a \in R$ and $m \in M$ satisfy $a m \in p M$. Suppose $s a \notin \sqrt{p}$. Set $K:=\left(p M:_{R} s m\right)$. Assume that $K \neq R$. Then there exists a maximal ideal $Q$ of $R$ so that $K \subseteq Q . m \notin T_{Q}(M)$, since otherwise, there exists $q \in Q$ such that $(1-q) m=0$ and so $(1-q) s m=0$. This implies that $(1-q) \in K \subseteq Q$, a contradiction. Since $M$ is $Q$-cyclic, by [4, Theorem 1.2], there exist $m^{\prime} \in M$ and 
$q \in Q$ such that $(1-q) M \subseteq R m^{\prime}$. In particular, $(1-q) m=s^{\prime} m^{\prime},(1-q) a m=p^{\prime} m^{\prime}$ for some $s^{\prime} \in R$ and $p^{\prime} \in p$. Thus $\left(a s^{\prime}-p^{\prime}\right) m^{\prime}=0$. Now $(1-q)\left(A n n_{R}\left(m^{\prime}\right)\right) M \subseteq$ $\left(A n n_{R}\left(m^{\prime}\right)\right) R m^{\prime}=\mathbf{0}$ implies $(1-q) A n n_{R}(m) \subseteq A n n_{R}(M)=\mathbf{0}$, because $M$ is faithful, and hence $(1-q) a s^{\prime}=(1-q) p^{\prime} \in p$. As $p$ is an $S$-primary ideal, $s s^{\prime} \in p$ or $s a \in \sqrt{p}$ or $s(1-q)^{n} \in p$ for some $n \in \mathbb{N}$. But $p \subseteq K \subseteq Q$ so that in each case, we have a contradiction. It follows that $K=R$ and $s m \in p M$, as required.

In the following, the Theorem 2.11 in [11] will be extended by removing the condition "finitely generated".

Theorem 2.17. Let $M$ be a multiplication $R$-module and $P$ a submodule of $M$ provided that $\left(P:_{R} M\right) \cap S=\emptyset$, where $S$ is a multiplicatively closed subset of $R$. Then the following are equivalent:

(a) $P$ is an $S$-primary (resp. S-prime) submodule of $M$.

(b) $\left(P:_{R} M\right)$ is an $S$-primary (resp. $S$-prime) ideal of $R$.

(c) $P=I M$ for some $S$-primary (resp. $S$-prime) ideal I of $R$ with $A n n(M) \subseteq$ $I$.

Proof. (a) $\Rightarrow$ (b) It is clear from Proposition 2.11 (a).

(b) $\Rightarrow$ (c) It is clear.

(c) $\Rightarrow$ (a) As $M$ is a faithful multiplication $R / A n n_{R}(M)$-module, by Corollary 2.10 (b), $I / A n n_{R}(M)$ is an $S$-primary (resp. $S$-prime) ideal of $R / A n n_{R}(M)$. Hence $P=I M$ is an $S$-primary (resp. $S$-prime) submodule of $R / A n n_{R}(M)$-module $M$ by Lemma 2.16. Therefore, $P$ is an $S$-primary (resp. $S$-prime) submodule of $R$-module $M$, as required.

Proposition 2.18. Let $P$ be an $S$-primary submodule of multiplication $R$-module $M$. Suppose that $N \cap L \subseteq P$ for some submodules $N$ and $L$ of $M$. Then $s N \subseteq P$ or $s L \subseteq \operatorname{rad}(P)$ for some $s \in S$.

Proof. Since $P$ is an $S$-primary submodule, there exists $s \in S$ such that for every $r \in R$ and $m \in M$, if $r m \in P$, then $s r \in \sqrt{\left(P:_{R} M\right)}$ or $s m \in P$. Let $s N \nsubseteq P$. Then $s m^{\prime} \notin P$ for some $m^{\prime} \in N$. Take an element $a \in\left(L:_{R} M\right)$. This yields that $a m^{\prime} \in\left(L:_{R} M\right) N \subseteq L \cap N \subseteq P$. As $P$ is an $S$-primary submodule of $M$ and $s m^{\prime} \notin P$, we can conclude that $s a \in \sqrt{\left(P:_{R} M\right)}$ so that $s\left(L:_{R} M\right) \subseteq \sqrt{\left(P:_{R} M\right)}$. As $M$ is a multiplication module, by [4, Theorem 2.12], we have

$$
s L=s\left(L:_{R} M\right) M \subseteq \sqrt{\left(P:_{R} M\right)} M=\operatorname{rad}(P) .
$$

Lemma 2.19. Let $R=R_{1} \times R_{2}$ and $S=S_{1} \times S_{2}$ where $S_{i}$ is a multiplicatively closed subset of $R_{i}$. Suppose $p=p_{1} \times p_{2}$ is an ideal of $R$. Then the following are equivalent: 
(a) $p$ is an $S$-primary ideal of $R$.

(b) $p_{1}$ is an $S_{1}$-primary ideal of $R_{1}$ and $p_{2} \cap S_{2} \neq \emptyset$ or $p_{2}$ is an $S_{2}$-primary ideal of $R_{2}$ and $p_{1} \cap S_{1} \neq \emptyset$.

Proof. $(\mathrm{a}) \Rightarrow(\mathrm{b})$ Since $(1,0)(0,1)=(0,0) \in p$, there exists $s=\left(s_{1}, s_{2}\right) \in S$ so that $s(1,0)=\left(s_{1}, 0\right) \in \sqrt{p}$ or $s(0,1)=\left(0, s_{2}\right) \in p$. Thus $p_{1} \cap S_{1} \neq \emptyset$ or $p_{2} \cap S_{2} \neq \emptyset$. We may assume that $p_{1} \cap S_{1} \neq \emptyset$. As $P \cap S=\emptyset$, we have $p_{2} \cap S_{2}=\emptyset$. Let $x y \in p_{2}$ for some $x, y \in R_{2}$. Since $(0, x)(0, y) \in p$ and $p$ is an $S$-primary ideal of $R$. We get either $s(0, x)=\left(0, s_{2} x\right) \in \sqrt{p}$ or $s(0, y)=\left(0, s_{2} y\right) \in p$ and this yields $s_{2} x \in \sqrt{p_{2}}$ or $s_{2} y \in p_{2}$. Therefore, $p_{2}$ is an $S$-primary ideal of $R_{2}$. In the other case, one can easily show that $p_{1}$ is an $S$-primary ideal of $R_{1}$.

(b) $\Rightarrow$ (a) Assume that $p_{1} \cap S_{1} \neq \emptyset$ and $p_{2}$ is an $S$-primary ideal of $R_{2}$. Then there exists $s_{1} \in p_{1} \cap S_{1}$. Let $(a, b)(c, d)=(a c, b d) \in p$ for some $a, c \in R_{1}$ and $b, d \in R_{2}$. This yields that $b d \in p_{2}$ and thus there exists $s_{2} \in S_{2}$ so that $s_{2} b \in \sqrt{p_{2}}$ or $s_{2} d \in p_{2}$. Put $s=\left(s_{1}, s_{2}\right) \in S$. Then note that $s(a, b)=\left(s_{1} a, s_{2} b\right) \in \sqrt{p}$ or $s(c, d) \in p$. Therefore, $p$ is an $S$-primary ideal of $R$. In other case, one can similarly prove that $p$ is an $S$-primary ideal of $R$.

Theorem 2.20. Suppose that $M=M_{1} \times M_{2}$ and $R=R_{1} \times R_{2}$-module and $S=S_{1} \times S_{2}$ is a multiplicatively closed subset of $R$, where $M_{i}$ is a $R_{i}$-module and $S_{i}$ is a multiplicatively closed subset of $R_{i}$ for each $i=1,2$. Assume $P=P_{1} \times P_{2}$ is a submodule of $M$. Then the following are equivalent:

(a) $P$ is an $S$-primary submodule of $M$.

(b) $P_{1}$ is an $S_{1}$-primary submodule of $M_{1}$ and $\left(P_{2}:_{R_{2}} M_{2}\right) \cap S_{2} \neq \emptyset$ or $P_{2}$ is an $S_{2}$-primary submodule of $M_{2}$ and $\left(P_{1}:_{R_{1}} M_{1}\right) \cap S_{1} \neq \emptyset$.

Proof. $(\mathrm{a}) \Rightarrow$ (b) By Proposition 2.11, $\left(P:_{R} M\right)=\left(P_{1}:_{R_{1}} M_{1}\right) \times\left(P_{2}:_{R_{2}} M_{2}\right)$ is an $S$-primary ideal of $R$ and so by Lemma 2.19, either $\left(P_{1}:_{R_{1}} M_{1}\right) \cap S_{1} \neq \emptyset$ or $\left(P_{2}: R_{2} M_{2}\right) \cap S_{2} \neq \emptyset$. We may assume that $\left(P_{1}:_{R_{1}} M_{1}\right) \cap S_{1} \neq \emptyset$. Now we will show that $P_{2}$ is an $S_{2}$-primary submodule of $M_{2}$. Let $r m \in P_{2}$ for some $r \in R_{2}$ and $m \in M_{2}$. Then $(1, r)(0, m)=(0, r m) \in P$. As $P$ is an $S$-primary, there is an $s=\left(s_{1}, s_{2}\right) \in S$ so that $s(1, r)=\left(s_{1}, s_{2} r\right) \in \sqrt{\left(P:_{R} M\right)}$ or $s\left(0:_{R} m\right)=\left(0, s_{2} m\right) \in$ $P$. This implies that $s_{2} r \in \sqrt{\left(P_{2}: R_{2} M_{2}\right)}$ or $s_{2} m \in P_{2}$. Therefore, $P_{2}$ is an $S_{2}$ is an $S_{2}$-primary submodule of $M_{2}$. In the other case, it can be similarly show that $P_{1}$ is an $S_{1}$-primary submodule of $M_{1}$.

(b) $\Rightarrow$ (a) Assume that $\left(P_{1}: R_{1} M_{1}\right) \cap S_{1} \neq \emptyset$ and $P_{2}$ is an $S_{2}$-primary submodule of $M_{2}$. Then there exists $s_{1} \in\left(P_{1}:_{R_{1}} M_{1}\right) \cap S_{1}$. Let $\left(r_{1}, r_{2}\right)\left(m_{1}, m_{2}\right)=\left(r_{1} m_{1}, r_{2} m_{2}\right) \in$ $P$ for some $r_{i} \in R_{i}$ and $m_{i} \in M_{i}$, where $i=1,2$. Then $r_{2} m_{2} \in P_{2}$. As $P_{2}$ is an $S_{2}$-primary submodule of $M_{2}$, there is an $s_{2} \in S_{2}$ so that $s_{2} r_{2} \in \sqrt{\left(P_{2}: R_{2} M_{2}\right)}$ or 
$s_{2} m_{2} \in P_{2}$. Now put $s=\left(s_{1}, s_{2}\right) \in S$. Then note that $s\left(r_{1}, r_{2}\right)=\left(s_{1} r_{1}, s_{2} r_{2}\right) \in$ $\sqrt{\left(P:_{R} M\right)}$ or $s\left(m_{1}, m_{2}\right)=\left(s_{1} m_{1}, s_{2} m_{2}\right) \in P_{1} \times P_{2}=P$. Therefore, $P$ is an $S$-primary submodule of $M$. Similarly, one can show that if $P_{1}$ is an $S_{1}$-primary submodule of $M_{1}$ and $\left(P_{2}:_{R_{2}} M_{2}\right) \cap S_{2} \neq \emptyset$, then $P$ is an $S$-primary submodule of $M$.

Theorem 2.21. Let $M=M_{1} \times M_{2} \times \cdots \times M_{n}$ and $R=R_{1} \times R_{2} \times \cdots \times R_{n}$-module and $S=S_{1} \times S_{2} \times \cdots \times S_{n}$ is a multiplicatively closed subset of $R$, where $M_{i}$ is an $R_{i}$-module and $S_{i}$ is a multiplicatively closed subset of $R_{i}$ for each $i=1,2, \ldots, n$. Assume $P=P_{1} \times P_{2} \times \cdots \times P_{n}$ is a submodule of $M$. Then the following are equivalent:

(a) $P$ is an $S$-primary submodule of $M$.

(b) $P_{i}$ is an $S_{i}$-primary submodule of $M_{i}$ for some $i \in\{1,2, \ldots, n\}$ and $\left(P_{j}:_{R_{j}}\right.$ $\left.M_{j}\right) \cap S_{j} \neq \emptyset$ for all $j \in\{1,2, \ldots, n\}-\{i\}$.

Proof. We apply induction on $n$. For $n=1$, the result is true. If $n=2$, then $(\mathrm{a}) \Leftrightarrow$ (b) follows from Theorem 2.20. Assume that (a) and (b) are equivalent when $k<n$. Now, we shall prove (a) $\Leftrightarrow$ (b) when $k=n$. Let $P=P_{1} \times P_{2} \times \cdots \times P_{n}$. Put $P^{\prime}=P_{1} \times P_{2} \times \cdots \times P_{n-1}$ and $S^{\prime}=S_{1} \times S_{2} \times \cdots \times S_{n-1}$. Then by Theorem 2.20, the necessary and sufficient condition for $P=P^{\prime} \times P_{n}$ is an $S$-primary submodule of $M$ is that $P^{\prime}$ is an $S$-primary submodule of $M^{\prime}$ and $\left(P_{n}:_{R_{n}} M_{n}\right) \cap S_{n} \neq \emptyset$ or $P_{n}$ is an $S$-primary submodule of $M_{n}$ and $\left(P^{\prime}: R^{\prime} M^{\prime}\right) \cap S^{\prime} \neq \emptyset$, where $M^{\prime}=$ $M_{1} \times M_{2} \times \cdots \times M_{n-1}$ and $R^{\prime}=R_{1} \times R_{2} \times \cdots \times R_{n-1}$. The rest follows from the induction hypothesis.

Lemma 2.22. Suppose that $P$ is an $S$-primary submodule of $M$. Then the following statements hold for some $s \in S$.

(a) $\left(P:_{M} s^{\prime}\right) \subseteq\left(P:_{M} s\right)$ for all $s^{\prime} \in S$.

(b) $\left(\left(P:_{R} M\right):_{R} s^{\prime}\right) \subseteq\left(\left(P:_{R} M\right):_{R} s\right)$ for all $s^{\prime} \in S$.

Proof. (a) Take an element $m^{\prime} \in\left(P:_{M} s^{\prime}\right)$, where $s^{\prime} \in S$. Then $s^{\prime} m^{\prime} \in P$. Since $P$ is an $S$-primary submodule of $M$, there exists $s \in S$ such that $s s^{\prime} \in \sqrt{\left(P:_{R} M\right)}$ or $s m^{\prime} \in P$. As $\left(P:_{R} M\right) \cap S=\emptyset$, we get $s m^{\prime} \in P$, namely $m^{\prime} \in\left(P:_{M} s\right)$.

(b) Follows from part (a).

Proposition 2.23. Suppose that $M$ is a finitely generated $R$-module, $S$ is a multiplicatively closed subset of $R$, and $P$ is a submodule of $M$ satisfying $\left(P:_{R} M\right) \cap S=$ $\emptyset$. Then the following are equivalent:

(a) $P$ is an $S$-primary submodule of $M$. 
(b) $S^{-1} P$ is a primary submodule of $S^{-1} M$ and there is an $s \in S$ satisfying $\left(P:_{M} s^{\prime}\right) \subseteq\left(P:_{M} s\right)$ for all $s^{\prime} \in S$.

Proof. $(\mathrm{a}) \Rightarrow(\mathrm{b})$ It is clear from Proposition 2.7 (c) and Lemma 2.22.

(b) $\Rightarrow\left(\right.$ a) Take $a \in R$ and $m \in M$ with $a m \in P$. Then $\frac{a}{1} \cdot \frac{m}{1} \in S^{-1} P$. Since $S^{-1} P$ is a primary submodule of $S^{-1} M$ and $M$ is finitely generated, we can conclude that $\frac{a}{1} \in \sqrt{\left(S^{-1} P:_{S^{-1} R} S^{-1} M\right)}=\sqrt{S^{-1}\left(P:_{R} M\right)}$ or $\frac{m}{1} \in S^{-1} P$. Then $u a \in$ $\sqrt{\left(P:_{R} M\right)}$ or $u^{\prime} m \in P$ for some $u, u^{\prime} \in S$. By assumption, there is an $s \in S$ so that $\left(P:_{R} s^{\prime}\right) \subseteq\left(P:_{R} s\right)$ for all $s^{\prime} \in S$. If $u a \in \sqrt{\left(P:_{R} M\right)}$, then $a^{n} M \subseteq\left(P:_{M}\right.$ $\left.u^{n}\right) \subseteq\left(P:_{R} s\right)$ for some $n \in \mathbb{N}$ and thus $s a \in \sqrt{\left(P:_{R} M\right)}$. If $u^{\prime} m \in P$, a similar argument shows that $s m \in P$. Therefore, $P$ is an $S$-primary submodule of $M$.

Theorem 2.24. Suppose that $P$ is a submodule of $M$ provided $\left(P:_{R} M\right) \cap S=\emptyset$. Then $P$ is an $S$-primary submodule of $M$ if and only if $\left(P:_{M} s\right)$ is a primary submodule of $M$ for some $s \in S$.

Proof. Assume $\left(P:_{M} s\right)$ is a primary submodule of $M$ for some $s \in S$. Let $a m \in P$, where $a \in R$ and $m \in M$. As $a m \in\left(P:_{M} s\right)$, we get $a \in \sqrt{\left(\left(P:_{M} s\right):_{R} M\right)}$ or $m \in$ $\left(P:_{M} s\right)$. This yields that $a s \in \sqrt{\left(P:_{R} M\right)}$ or $s m \in P$. Conversely, assume that $P$ is an $S$-primary submodule of $M$. Then there exists $s \in S$ such that whenever $a m \in P$, where $a \in R$ and $m \in M$, then $s a \in \sqrt{\left(P:_{R} M\right)}$ or $s m \in P$. Now we prove that $\left(P:_{M} s\right)$ is primary. Take $r \in R$ and $m \in M$ with $r m \in\left(P:_{M} s\right)$. Then $s r m \in P$. As $P$ is $S$-primary, we get $s^{2} r \in \sqrt{\left(P:_{R} M\right)}$ or $s m \in P$. If $s m \in P$, then there is nothing to show. Assume that $s m \notin P$. Then $s^{2} r \in \sqrt{\left(P:_{R} M\right)}$ and hence $s r \in \sqrt{\left(P:_{R} M\right)}$. Thus $r^{n} \in\left(\left(P:_{R} M\right):_{R} s^{n}\right) \subseteq\left(\left(P:_{R} M\right):_{R} s\right)$ for some $n \in \mathbb{N}$, by Lemma 2.22. Thus, we can conclude that $r^{n} \in\left(\left(P:_{M} s\right):_{R} M\right)$, namely $r \in \sqrt{\left(\left(P:_{M} s\right):_{R} M\right)}$. Hence $\left(P:_{M} s\right)$ is a prime submodule of $M$.

Theorem 2.25. Suppose that $P$ is a submodule of $M$ provided $\left(P:_{R} M\right) \subseteq \operatorname{Jac}(R)$, where $\operatorname{Jac}(R)$ is the Jacobson radical of $R$. Then the following statements are equivalent:

(a) $P$ is a primary submodule of $M$.

(b) $\left(P:_{R} M\right)$ is a primary ideal of $R$ and $P$ is an $(R-\mathfrak{m})$-primary submodule of $M$ for each $\mathfrak{m} \in \operatorname{Max}(R)$.

Proof. $(\mathrm{a}) \Rightarrow(\mathrm{b})$ Since $\left(P:_{R} M\right) \subseteq \operatorname{Jac}(R),\left(P:_{R} M\right) \subseteq \mathfrak{m}$ for each $\mathfrak{m} \in \operatorname{Max}(R)$ and hence $\left(P:_{R} M\right) \cap(R-\mathfrak{m})=\emptyset$. The rest follows from Lemma 2.3 (a).

(b) $\Rightarrow(\mathrm{a})$ Let $a m \in P$ with $a \notin\left(P:_{R} M\right)$ for some $a \in R$ and $m \in M$. Let $\mathfrak{m} \in \operatorname{Max}(R)$. As $P$ is an $(R-\mathfrak{m})$-primary submodule of $M$, there exists $s_{\mathfrak{m}} \notin \mathfrak{m}$ such that $a s_{\mathfrak{m}} \in \sqrt{\left(P:_{R} M\right)}$ or $s_{\mathfrak{m}} m \in P$. As $\left(P:_{R} M\right)$ is a primary ideal of $R$ and 
$s_{\mathfrak{m}} \notin \sqrt{\left(P:_{R} M\right)}$, we have $a s_{\mathfrak{m}} \notin\left(P:_{R} M\right)$ and so $s_{\mathfrak{m}} m \in P$. Now consider the set $\Omega=\left\{s_{\mathfrak{m}} \mid \exists \mathfrak{m} \in \operatorname{Max}(R), s_{\mathfrak{m}} \notin \mathfrak{m}\right.$ and $\left.s_{\mathfrak{m}} m \in P\right\}$. Then note that $(\Omega)=R$. To see this, take any maximal ideal $\mathfrak{m}^{\prime}$ containing $\Omega$. Then the definition of $\Omega$ requires that there exists $s_{\mathfrak{m}^{\prime}} \in \Omega$ and $s_{\mathfrak{m}^{\prime}} \notin \mathfrak{m}^{\prime}$. As $\Omega \subseteq \mathfrak{m}^{\prime}$, we have $s_{\mathfrak{m}^{\prime}} \in \Omega \subseteq \mathfrak{m}^{\prime}$, a contradiction. Thus $(\Omega)=R$ and this yields $1=r_{1} s_{\mathfrak{m}_{1}}+r_{2} s_{\mathfrak{m}_{2}}+\cdots+r_{n} s_{\mathfrak{m}_{n}}$ for some $r_{i} \in R$ and $s_{\mathfrak{m}_{i}} \notin \mathfrak{m}_{i}$ with $s_{\mathfrak{m}_{i}} m \in P$, where $\mathfrak{m}_{i} \in \operatorname{Max}(R)$ for each $i=1,2, \ldots, n$. This yields that $m=r_{1} s_{\mathfrak{m}_{1}} m+r_{2} s_{\mathfrak{m}_{2}} m+\cdots+r_{n} s_{\mathfrak{m}_{n}} m \in P$. Therefore, $P$ is a primary submodule of $M$.

Now we determine all primary submodules of a module over a quasi-local ring in terms of $S$-primary submodules.

Corollary 2.26. Suppose $M$ is a module over a quasi-local ring $(R, \mathfrak{m})$. Then the following statements are equivalent:

(a) $P$ is a primary submodule of $M$.

(b) $\left(P:_{R} M\right)$ is a primary ideal of $R$ and $P$ is an $(R-\mathfrak{m})$-primary submodule of $M$ for each $\mathfrak{m} \in \operatorname{Max}(R)$.

Proof. This is clear from Theorem 2.25.

Remark 2.27. (a) Suppose that $M$ is an $R$-module. The idealization $R(+) M$ $=\{(a, m) \mid a \in R, m \in M\}$ of $M$ is a commutative ring whose addition is component-wise and whose multiplication is defined as $(a, m)\left(b, m^{\prime}\right)=$ $\left(a b, a m^{\prime}+b m\right)$ for each $a, b \in R$ and $m, m^{\prime} \in M$. If $S$ is a multiplicatively closed subset of $R$ and $P$ is a submodule of $M$, then $S(+) P=\{(s, p) \mid s \in$ $S, p \in P\}$ is a multiplicatively closed subset of $R(+) M[2,10]$.

(b) Radical ideals of $R(+) M$ have the form $I(+) M$, where $I$ is a radical ideal of $R$. If $J$ is an ideal of $R(+) M$, then $\sqrt{J}=\sqrt{I}(+) M$. In particular, if $I$ is an ideal of $R$ and $N$ is a submodule of $M$, then $\sqrt{I(+) N}=\sqrt{I}(+) M[2$, Theorem $3.2(3)]$.

Proposition 2.28. Let $M$ be an $R$-module and $p$ be an ideal of $R$ such that $p \subseteq$ Ann $(M)$. Then the following are equivalent:

(a) $p$ is a primary ideal of $R$.

(b) $p(+) M$ is a primary ideal of $R(+) M$.

Proof. This is straightforward.

Theorem 2.29. Let $S$ be a multiplicatively closed subset of $R, p$ be an ideal of $R$ provided $p \cap S=\emptyset$ and $M$ be an $R$-module. Then the following are equivalent:

(a) $p$ is an $S$-primary ideal of $R$. 
(b) $p(+) M$ is an $S(+) 0$-primary ideal of $R(+) M$.

(c) $p(+) M$ is an $S(+) M$-primary ideal of $R(+) M$.

Proof. $(\mathrm{a}) \Rightarrow(\mathrm{b})$ Let $(x, m)\left(y, m^{\prime}\right)=\left(x y, x m^{\prime}+y m\right) \in p(+) M$, where $x, y \in R$ and $m, m^{\prime} \in M$. Then we get $x y \in p$. As $p$ is $S$-primary, there exists $s \in S$ such that $s x \in \sqrt{p}$ or $s y \in p$. Now put $s^{\prime}=(s, 0) \in S(+) 0$. Then we have $s^{\prime}(x, m)=(s x, s m) \in \sqrt{p}(+) M=\sqrt{p(+) M}$ or $s^{\prime}\left(y, m^{\prime}\right)=\left(s y, s m^{\prime}\right) \in p(+) M$. Therefore, $p(+) M$ is an $S(+) 0$-primary ideal of $R(+) M$.

(b) $\Rightarrow$ (c) It is clear from Proposition 2.7.

$(\mathrm{c}) \Rightarrow(\mathrm{a})$ Let $x y \in p$ for some $x, y \in R$. Then $(x, 0)(y, 0) \in p(+) M$. Since $p(+) M$ is $S(+) M$-primary, there exists $s=\left(s_{1}, m_{1}\right) \in S(+) M$ such that $s(x, 0)=$ $\left(s_{1} x, x m_{1}\right) \in \sqrt{p(+) M}=\sqrt{p}(+) M$ or $s(y, 0)=\left(s_{1} y, y m_{1}\right) \in p(+) M$ and hence we get $s_{1} x \in \sqrt{p}$ or $s_{1} y \in p$. Therefore $p$ is an $S$-primary ideal of $R$.

Remark 2.30. Let $M$ be an $R$-module and let $S$ be a multiplicatively closed subset of $R$ such that $A n n_{R}(M) \cap S=\emptyset$. We say that $M$ is an $S$-torsion-free module in the case that there is an $s \in S$ such that if $r m=0$, where $r \in R$ and $m \in M$, then $s m=0$ or $s r=0[11$, Definition 2.23].

Proposition 2.31. Let $M$ be an $R$-module. Assume that $P$ is a submodule of $M$ and $S$ is a multiplicatively closed subset of $R$ such that $A n n_{R}(M) \cap S=\emptyset$. Then $P$ is an $S$-primary submodule of $M$ if and only if the factor module $M / P$ is a $\pi(S)$ torsion-free $R / \sqrt{\left(P:_{R} M\right)}$-module, where $\pi: R \rightarrow R / \sqrt{\left(P:_{R} M\right)}$ is the canonical homomorphism.

Proof. Suppose that $P$ is an $S$-primary submodule of $M$. Let $\overline{a m}=0_{M / P}$, where $\bar{a}=a+\sqrt{\left(P:_{R} M\right)}$ and $\bar{m}=m+P$ for some $a \in R$ and $m \in M$. This yields that $a m \in P$. As $P$ is $S$-primary, there exists $s \in S$ such that $s a \in \sqrt{\left(P:_{R} M\right)}$ or $s m \in$ $P$. Then we can conclude that $\pi(s) \bar{a}=0_{R / \sqrt{\left(P:_{R} M\right)}}$ or $\pi(s) \bar{m}=0_{M / P}$. Therefore, $M / P$ is a $\pi(S)$-torsion-free $R / \sqrt{\left(P:_{R} M\right)}$-module. For the other direction, suppose that $M / P$ is a $\pi(S)$-torsion-free $R / \sqrt{\left(P:_{R} M\right)}$-module. Let $a m \in P$, where $a \in R$ and $m \in M$. Put $\bar{a}=a+\sqrt{\left(P:_{R} M\right)}$ and $\bar{m}=m+P$. Then note that $\overline{a m}=0_{M / P}$. As $M / P$ is a $\pi(S)$-torsion-free $R / \sqrt{\left(P:_{R} M\right)}$-module, there exists $s \in S$ such that $\pi(s) \bar{a}=0_{R / \sqrt{\left(P:_{R} M\right)}}$ or $\pi(s) \bar{m}=0_{M / P}$. This yields that $s a \in \sqrt{\left(P:_{R} M\right)}$ or $s m \in P$. Accordingly, $P$ is an $S$-primary submodule of $M$.

Definition 2.32. Let $M$ be an $R$-module and let $S$ be a multiplicatively closed subset of $R$ such that $A n n_{R}(M) \cap S=\emptyset$. We say that $M$ is a quasi $S$-torsion-free module, if there exists $s \in S$ such that whenever $r m=0$, where $r \in R$ and $m \in M$, then $s m=0$ or $(s r)^{t}=0$ for some $t \in \mathbb{N}$. 
According to Definition 2.32, Proposition 2.31 can be expressed as follows.

Proposition 2.33. Let $M$ be an $R$-module. Assume that $P$ is a submodule of $M$ and $S$ is a multiplicatively closed subset of $R$ such that $A n n_{R}(M) \cap S=\emptyset$. Then $P$ is an $S$-primary submodule of $M$ if and only if the factor module $M / P$ is a quasi $\pi^{\prime}(S)$-torsion-free $R /\left(P:_{R} M\right)$-module, where $\pi^{\prime}: R \rightarrow R /\left(P:_{R} M\right)$ is the canonical homomorphism.

Theorem 2.34. Let $M$ be a module over an integral domain $R$. The following are equivalent:

(a) $M$ is a torsion-free module;

(b) $M$ is a quasi $(R-p)$-torsion-free module for each $p \in \operatorname{Spec}(R)$;

(c) $M$ is a quasi $(R-\mathfrak{m})$-torsion-free module for each $\mathfrak{m} \in \operatorname{Max}(R)$.

Proof. $(\mathrm{a}) \Rightarrow(\mathrm{b})$ It is clear.

$(\mathrm{b}) \Rightarrow(\mathrm{c})$ It is clear.

(c) $\Rightarrow$ (a) Assume that $a \neq 0$. Take $\mathfrak{m} \in \operatorname{Max}(R)$. As $M$ is quasi $(R-\mathfrak{m})$-torsionfree, there exists $s_{m} \neq \mathfrak{m}$ so that $s_{m} m=0$ or $\left(s_{m} a\right)^{t}=0$ for some $t \in \mathbb{N}$. As $R$ is an integral domain, $\left(s_{m} a\right)^{t} \neq 0$. Now, put $\Omega=\left\{s_{m} \in R \mid \exists \mathfrak{m} \in \operatorname{Max}(R), s_{m} \notin\right.$ $\mathfrak{m}$ and $\left.s_{m} m=0\right\}$. A similar argument in the proof of Theorem 2.25 shows that $\Omega=R$. Then we have $\left(s_{m_{1}}\right)+\left(s_{m_{2}}\right)+\cdots+\left(s_{m_{n}}\right)=R$ for some $\left(s_{m_{i}}\right) \in \Omega$. This implies that $R m=\sum_{i=1}^{n}\left(s_{m_{i}}\right) m=(0)$ and hence $m=0$. This means $M$ is a torsion-free module.

Acknowledgement. The authors would like to thank the referees for their valuable comments. Also, we would like to thank professor A. Harmanci for his helpful advice on this work.

\section{References}

[1] R. Ameri, On the prime submodules of multiplication modules, Int. J. Math. Math. Sci., 27 (2003), 1715-1724.

[2] D. D. Anderson and M. Winders, Idealization of a module, J. Commut. Algebra, 1(1) (2009), 3-56.

[3] J. Dauns, Prime modules, J. Reine Angew. Math., 298 (1978), 156-181.

[4] Z. A. El-Bast and P. F. Smith, Multiplication modules, Comm. Algebra, 16(4) (1988), 755-779.

[5] F. Farshadifar, S-secondary submodules of a module, Comm. Algebra, 49(4) (2021), 1394-1404. 
[6] R. Gilmer, Multiplicative Ideal Theory, Queen's Papers in Pure and Applied Mathematics, 90, Queen's University, Kingston, 1992.

[7] C. P. Lu, A module whose prime spectrum has the surjective natural map, Houston J. Math., 33(1) (2007), 125-143.

[8] R. L. McCasland and M. E. Moore, On radicals of submodules of finitely generated modules, Canad. Math. Bull., 29(1) (1986), 37-39.

[9] R. L. McCasland and M. E. Moore, Prime submodules, Comm. Algebra, 20(6) (1992), 1803-1817.

[10] M. Nagata, Local Rings, Interscience Tracts in Pure and Applied Mathematics, $13,1962$.

[11] E. Sevim, T. Arabaci, U. Tekir and S. Koc, On S-prime submodules, Turkish J. Math., 43(2) (2019), 1036-1046.

[12] F. Wang and H. Kim, Foundations of Commutative Rings and Their Modules, Algebra and Applications, 22, Springer, Singapore, 2016.

H. Ansari-Toroghy (Corresponding Author) and S. S. Pourmortazavi

Department of Pure Mathematics

Faculty of Mathematical Sciences

University of Guilan

P. O. Box 41335-19141 Rasht, Iran

e-mails: ansari@guilan.ac.ir (H. Ansari-Toroghy)

mortazavi@phd.guilan.ac.ir (S. S. Pourmortazavi) 